\title{
Estimation De L'exhaustivité De La Surveillance Des Intoxications Médicamenteuses Dans La Région De Tanger-Tétouan-Al Hoceima, Maroc, 2014-2016
}

\author{
Soumaia Hmimou, \\ Hinde Hami,
}

PPR-B-Mokhtari-FS-UIT-Kenitra, Laboratoire de Génétique et Biométrie, Faculté des Sciences, Université Ibn Tofail, Kénitra, Maroc

\section{Rachid Hmimou,}

Centre Anti Poison et de Pharmacovigilance du Maroc, Rabat, Maroc

\section{Abdelmajid Soulaymani, Abdelrhani Mokhtari,}

PPR-B-Mokhtari-FS-UIT-Kenitra, Laboratoire de Génétique et Biométrie, Faculté des Sciences, Université Ibn Tofail, Kénitra, Maroc

\section{Rachida Soulaymani-Bencheikh,}

Centre Anti Poison et de Pharmacovigilance du Maroc, Rabat, Maroc

Faculté de Médecine et de Pharmacie, Université Mohammed V, Rabat, Maroc

\section{Doi: 10.19044/esj.2018.v14n12p78 URL:http://dx.doi.org/10.19044/esj.2018.v14n12p78}

\begin{abstract}
The aim of this study is to estimate the completeness of drug poisoning surveillance in the region of Tanger-Tétouan-Al Hoceima in Morocco. The study deals with cases of drug poisoning identified by the Moroccan Anti Poison Control Center (MPCC) and the cases collected by the registers of the provincial hospitals of the studied region between 2014 and 2016 period. The completeness of the surveillance was studied by the capture-recapture method. During the study period, 835 cases of drug poisoning were identified by the MPCC and 892 cases from the registers of hospitals in the region. The number of duplicates was 78. The capture-recapture method made it possible to estimate the total number of cases at 9549 (95\% CI: 8,199-10,900). The completeness of surveillance is estimated at $8.74 \%$ from MPCC and $9.34 \%$ from hospital registers in the region. The capture-recapture method provided limited completeness of monitoring for drug poisoning in the study area. Improved awareness of doctors about the declaration is necessary to strengthen the system of surveillance of poisoning in Morocco.
\end{abstract}


Keywords: Capture-recapture method, Completeness, Tanger-Tétouan-Al Hoceima, Morocco, MPCC, Registers of hospitals

\section{Résumé}

La présente étude vise à estimer l'exhaustivité de la surveillancedes intoxications médicamenteuses survenues dans la région de Tanger-TétouanAl Hoceima au Maroc. L'étude porte sur les cas d'intoxications médicamenteusesrecensés par le Centre Anti Poison et de Pharmacovigilance du Maroc (CAPM) et les cas collectés par les registres des hôpitaux provinciaux de la région étudiée durant la période 2014-2016. L'exhaustivitéde la surveillance a été étudiée par la méthode capturerecapture. Pendant la période d'étude, 835 cas d'intoxications par médicaments ont été recensés par le CAPM et 892 cas à partir des registres des hôpitaux de la région. Le nombre de doublons était de 78 . La méthode capture-recapture a permis d'estimer le nombre total de cas à 9549 (IC à 95\%: 8 199-10 900). Le taux d'exhaustivité de la surveillance est estimé à $8,74 \%$ à partir des données du CAPM et à 9,34\% à partir des registres des hôpitaux de la région. La méthode capture-recapture a donné une exhaustivité limitée de la surveillance des intoxications médicamenteuses dans la région étudiée. Une meilleure sensibilisation des médecins à la déclaration s'avère nécessaire afin de renforcer le système de surveillance des intoxications au Maroc.

Mots clés: Méthode capture-recapture, Exhaustivité, Tanger-Tétouan-A1 Hoceima, Maroc, CAPM, Registres des hôpitaux.

\section{Introduction}

Les intoxications médicamenteuses constituent un véritable problème de santé publique dans de nombreux pays. Elles constituent une des premières causes d'admission aux urgences et d'hospitalisation en réanimation médicale.

En effet, 50\% des appels reçus au Centre Anti-Poison du Québec, de 2008 à 2014, concernaient des intoxications potentielles par médicaments (Lebel G. \& Dubé M., 2015).

Une étude menée sur les cas d'intoxications survenus durant la période 1997-2002 chez l'adulte à Adana en Turquie a montré que 59\% des cas étaient dus à des médicaments (Seydaoglu G.et al., 2005).

Durant l'année 2002, le Centre Antipoison de Lille a enregistré 30712 cas d'intoxications humaines dont les médicaments sont les produits en cause dans 57\% des cas (Mathieu-Nolf M, 2002).

En 2015, le Centre Anti Poison et de Pharmacovigilance du Maroc (CAPM) a reçu 15290 cas d'intoxications dont les intoxications médicamenteuses occupent la première place, avec 29,8\% des cas (Hmimou R.et al., 2015). 
L'objectif de la présente étude est d'estimer le nombre total de cas etd'évaluerl'exhaustivité de la surveillance des intoxications médicamenteuses survenues entre Janvier 2014 et Décembre 2016 dans la région de Tanger-Tétouan-Al Hoceima au Maroc.

\section{Matériel et méthodes}

Il s'agit d'une étude descriptive des cas d'intoxications par médicaments enregistrés entre janvier 2014 et décembre 2016 dans la région de Tanger-Tétouan-Al Hoceima au Maroc.L'exhaustivitéde la surveillance des intoxications médicamenteuses a été étudiée par la méthode capture-recapture à 2 sources.

\section{Définition des cas}

Pour l'étude, on a retenu la définition de cas suivante: toutpatient intoxiqué par un ou plusieurs médicamentsau niveau de la région de TangerTétouan-Al Hoceima entre janvier 2014 et décembre 2016.

\section{Sources de données}

La première sourcepour cette étude était l'ensemble des cas d'intoxications médicamenteuses déclarés au CAPM, qui est une institution d'utilité publique parrainée par le Ministère de la Santé. Il s'occupe de la gestion des intoxications au Maroc et assure des missionsde veille, de vigilance et d'alerte sanitaire.

Depuis 1980, la déclaration de tous les cas d'intoxications est devenue obligatoire au Maroc (circulaire ministérielle $n^{\circ} 19$ 829DR/ BF/MM). Chaque préfecture et province devaient fournir au CAPM le relevé de tous les cas d'intoxications admis dans les structures sanitaires.

Khattabi A. et al., 2009)

La base de données nationale des intoxications est constituée desdonnées issues des appels reçus à l'unité de l'information toxicologique (réponse téléphonique) etdes fiches de déclarationdes intoxications reçuesà l'unité de toxicovigilance.

La deuxième source était l'ensemble des cas diagnostiqués par les 11 hôpitaux de la région d'étude (Hôpital Mohammed V à Tanger, hôpital Assilahà Assilah, hôpital civil à Tétouan, hôpital Mohammed V à Al Hoceima, hôpital Lalla Meriem à Larache, hôpital Mohammed V à Chefchaouen, hôpital Mohammed VI à M'diq, hôpital Hassan II à Fnideq, hôpital Abou Kacem Zahraoui à Ouezzane, hôpital Ksar El Kebir à Ksar El Kebir et hôpital de Martil).

Les principales informations recueillies par les deux sources sont : le type d'intoxication, la région, la province, la date de l'intoxication, le service d'admission, l'âge, le sexe, le lieu, le nom de(s)médicament(s), les signes 
cliniques, le traitement préconisé et l'évolution.

\section{Méthode capture-recapture à deux sources Principe}

La méthode capture-recapture à deux sources permet, en croisant les cas d'une maladie recensés par deux systèmes de surveillance A et $\mathrm{B}$, et après avoir identifié les cas communs entre les deux systèmes,d'estimer le nombre de cas identifiés par aucun des systèmes, le nombre total de cas et l'exhaustivité de chaque système International Working Group for Disease Monitoring and Forecasting, 1995; Van Hest R.,2007 ;Campèse C.\& Che D.,2012).

\section{Estimation du nombre total de cas avec deux sources}

Le croisement des cas identifiés par les deux sources de données est illustré par un tableau de contingence $2 \times 2$ (Tableau 1). Les cas sont répartis selon leur présence ou absence dans l'une ou l'autre source.

Tableau 1 : Tableaude contingence représentant le croisement de deux sources de données

\begin{tabular}{|c|c|c|c|c|}
\cline { 3 - 5 } \multicolumn{2}{c|}{} & \multicolumn{3}{c|}{ Source A } \\
\cline { 3 - 5 } \multicolumn{2}{c|}{} & Oui & Non & Total \\
\hline \multirow{3}{*}{ Source B } & Oui & $\mathrm{n}_{11}$ & $\mathrm{n}_{21}$ & $\mathrm{~N}_{\mathrm{B}}$ \\
\cline { 2 - 5 } & Non & $\mathrm{n}_{12}$ & $\mathrm{n}_{22}$ & - \\
\cline { 2 - 5 } & Total & $\mathrm{N}_{\mathrm{A}}$ & - & $\mathrm{N}$ \\
\hline
\end{tabular}

$\mathrm{N}_{\mathrm{A}}$ et $\mathrm{N}_{\mathrm{B}}$ : Nombre de cas notifiés dans chacune des sources, $\mathrm{N}$ : Nombre total de cas, à estimer, $\mathrm{n}_{11}$ : Nombre de cas communs aux sources (doublons), $\mathrm{n}_{22}$ : Nombre de cas non identifiés par les deux sources, à estimer.

Sous l'hypothèse d'indépendance des deux sources, Sekar et Demingproposent des estimateurs suivants (Cormack R.M., 1968 ; Sekar C.C.\& deming W.E., 1949)

Le nombre de cas non identifiés par les 2 sources :

$$
\mathrm{n}_{22}=\frac{\mathrm{n}_{12} \times \mathrm{n}_{21}}{\mathrm{n}_{11}}
$$

Le nombre total de cas, sa variance et son intervalle de confiance (IC) à 95\% :

$$
\begin{gathered}
\mathrm{N}=\frac{\mathrm{N}_{\mathrm{A}} \times \mathrm{N}_{\mathrm{B}}}{\mathrm{n}_{11}} \\
\operatorname{Var}(\mathrm{N})=\frac{\mathrm{N}_{\mathrm{A}} \times \mathrm{N}_{\mathrm{B}} \times \mathrm{n}_{12} \times \mathrm{n}_{21}}{\mathrm{n}_{11}{ }^{3}} \\
\operatorname{IC} 95 \%(\mathrm{~N})=\mathrm{N} \pm 1,96 \sqrt{\operatorname{Var}(\mathrm{N})}
\end{gathered}
$$


Chapman et Seber ont montré que ces estimateurs pouvaient être biaisés lorsque les effectifs sont faibles et que $\mathrm{n}_{11} \mathrm{a}$ une probabilité non nulle d'être égal à zéro. Ils ont proposé les estimateurs non biaisés suivants (Chapman D G, 1951 ;Seber G.A.F, 1970):

$$
\begin{gathered}
\mathrm{N}=\frac{\left(\mathrm{N}_{\mathrm{A}}+1\right)\left(\mathrm{N}_{\mathrm{B}}+1\right)}{\left(\mathrm{n}_{11}+1\right)}-1 \\
\operatorname{Var}(\mathrm{N})=\frac{\left(\mathrm{N}_{\mathrm{A}}+1\right)\left(\mathrm{N}_{\mathrm{B}}+1\right) \mathrm{n}_{12} \mathrm{n}_{21}}{\left(\mathrm{n}_{11}+1\right)^{2}\left(\mathrm{n}_{11}+2\right)}
\end{gathered}
$$

Les taux d'exhaustivité des deux sources sont :

Source A :

$$
\mathrm{E}_{\mathrm{A}}=\frac{\mathrm{N}_{\mathrm{A}}}{\mathrm{N}}=\frac{\mathrm{n}_{11}}{\mathrm{~N}_{\mathrm{B}}}
$$

Source B :

$$
E_{B}=\frac{N_{B}}{N}=\frac{n_{11}}{N_{A}}
$$

Celui des deux sources combinées est : $E_{A B}=\frac{N_{A}+N_{B}-n_{11}}{N}$

\section{Conditions d'application}

Les conditions d'application de la méthode capture-recapture sont : l'indépendance des deux sources, l'homogénéité de capture des cas, tous les cas identifiés sont des vrais cas, recensés sur la même période et la même zone géographique pour les deux sources, tous les vrais cas communs et seulement les vrais cas communs sont identifiés et la population étudiée est close (Hubert B.\&Desenclos J-C, 1993 ; Perrocheau A.\&Levy-Bruhl D., 2000)

L'indépendance entre les deux sources peut être vérifiée, selon Sekar et Deming (1949), par une analyse stratifiée sur des variables d'hétérogénéité de capture, par exemple par sexe. On calcule pour chacunedes strates i les taux d'exhaustivité de chaque source $\mathrm{E}_{\mathrm{Ai}}$ et $\mathrm{E}_{\mathrm{Bi}}$.L'indépendance est alors testée par le coefficient de corrélation $r$ entre ces taux $\mathrm{E}_{\mathrm{Ai}}$ et $\mathrm{E}_{\mathrm{Bi}}$, pondérés par $\mathrm{N}_{\mathrm{i}}$ nombre de cas estimés dans chaque strate(Bettaieb J.et al.,2013)..

$$
r=\frac{\sum N_{i}\left(E_{A i}-E_{A}\right)\left(E_{B i}-E_{B}\right)}{\sqrt{\sum N_{i}\left(E_{A i}-E_{A}\right)^{2} \sum N_{i}\left(E_{B i}-E_{B}\right)^{2}}}
$$

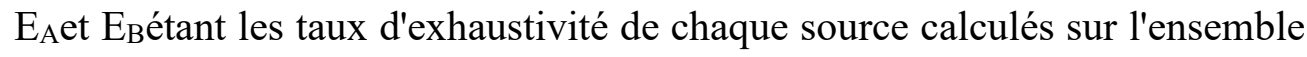
de la population non stratifiée.

La saisie des données a été faite avecle logiciel Epi Data.

\section{Résultats}

Durant la période 2014-2016, 835 cas d'intoxications 
médicamenteuses ont été déclarés au Centre Anti Poison et de Pharmacovigilance du Maroc (CAPM) et 892 cas ont été enregistrés dans les registres des hôpitaux de la région de Tanger-Tétouan-Al Hoceima (RHR).

Selon les résultats, la majorité des cas est de sexe féminin avec un sexeratio $(\mathrm{F} / \mathrm{M})$ de 2,21,d'après le CAPM et 3,4, d'après les RHR. L'âge moyen des intoxiqués est de 17,6 $\pm 14,48$ ans, suivant les données de la surveillanceet $24,08 \pm 12,21$ ans pour la source RHR. Les adolescents et les jeunes adultes 2029 ans sont les plus concernés, avec $43,11 \%$ des cas identifiés par la surveillance contre $65,44 \%$ des cas enregistrés dans la sourceRHR $\left(\mathrm{p}<10^{-3}\right)$.

La région a enregistré une augmentation du nombre de cas en 2015, d'après les deux sources.

Un maximum de $58 \%$ des cas a été enregistré dans les provinces de Tétouan et Tanger-Assilah pour les deux sources.

La proportion des cas d'intoxications par médicaments selon l'origine de l'intoxiqué différait significativement entre les deux systèmes $\left(\mathrm{p}<10^{-3}\right)$, avec une prédominance des casen milieu urbain.

En comparant la proportion de cas identifiés selon les circonstances de l'intoxication par source, on constate que dans la majorité des cas, les intoxications médicamenteuses sont accidentelles. Toutefois, on n'a pas noté de différence significative entre les deux systèmes $(\mathrm{p}=0,50)$.

L'évolution était favorable dansplus de $99 \%$ des cas, avec une différence non significative entre les deux systèmes $(p=0,61)$.

L'ensemble des variables principales était renseigné, en moyenne, pour 93,26\% des cas recensés par le Centre Anti Poison et de Pharmacovigilance (CAPM) et 85,49\% des cas collectés par les registres des hôpitaux de la région étudiée(RHR).

Tableau 2 : Pourcentage de cas renseignés pour chaque variable selon les sources, Tanger-

Tétouan-Al Hoceima, Maroc, 2014-2016

\begin{tabular}{|c|c|c|}
\hline \multicolumn{3}{|c|}{\begin{tabular}{|l} 
Pourcentage de cas renseignés (\%) \\
\end{tabular}} \\
\hline & CAPM & RHR \\
\hline Date de l'intoxication & 100 & 100 \\
\hline Ville & 100 & 100 \\
\hline Sexe & 98,08 & 95,63 \\
\hline Age & 95,57 & 75,89 \\
\hline Origine & 87,78 & 31,05 \\
\hline $\begin{array}{l}\text { Circonstances de } \\
\text { l'intoxication }\end{array}$ & 94,61 & 97,42 \\
\hline Evolution & 76,77 & 98,43 \\
\hline
\end{tabular}

\section{Recherche des cas communs}

La recherche des cas communs entre les deux sources a permis d'identifier 78 doublons (Figure 1). 


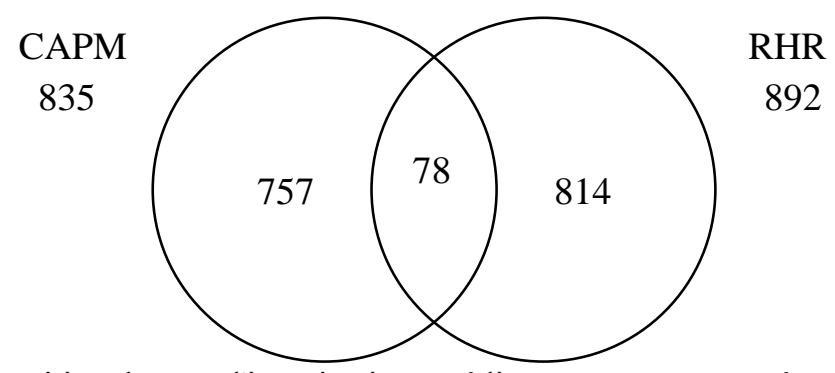

Figure 1 : Répartition des cas d'intoxications médicamenteuses recensés par deux sources

L'application de la méthode capture-recapture a permis d'estimer le nombre total de cas d'intoxications médicamenteuses entre 2014 et 2016 à 9 549 (IC à 95\%: 8 199-10 900).

Tableau 3 : Croisement des cas identifiés dans les deux sources, Tanger-Tétouan-Al Hoceima, Maroc, 2014-2016

\begin{tabular}{|c|c|c|c|c|}
\cline { 3 - 5 } \multicolumn{2}{c|}{} & \multicolumn{3}{c|}{ CAPM } \\
\cline { 3 - 5 } \multicolumn{2}{c|}{} & Oui & Non & Total \\
\hline \multirow{3}{*}{ RHR } & Oui & 78 & 814 & 892 \\
\cline { 2 - 5 } & Non & 757 & 7900 & 8657 \\
\cline { 2 - 5 } & Total & 835 & 8714 & 9549 \\
\hline
\end{tabular}

Le taux d'exhaustivité de la surveillance est estimé à $8,74 \%$ à partir des données du CAPM et à 9,34\% à partir des registres des hôpitaux de la région. Le regroupement des deux sources permet de recenser $17,26 \%$ du nombre total de cas.

\section{Indépendance des sources}

Le test de corrélation entreles taux de déclaration de chacunedes deux sourcespar année ne met pas en évidence de dépendance entre les deux sources $\left(\mathrm{r}=0, \mathrm{p}<10^{-3}\right)$.La probabilité qu'un cas soit notifié dans l'une des deux sources ne soit pas affectée par la probabilité qu'il soit notifié dans l'autre.

L'analyse stratifiée sur chacune des variables susceptibles d'entraîner une hétérogénéité de capture des casne montre pas de différence entre l'estimation totale du nombre de caset la somme des estimations par stratepour l'année de déclaration(9 549 cas), la province(9 549 cas), l'âge (8 752 cas), le sexe (8 957 cas) et les circonstances de l'intoxication(8 801 cas)(Tableau 4). Chacune des sommes des estimations stratifiées étant comprise dans l'IC 95\% de l'estimation globale entre 8199 et 10900 cas.

Toutefois, la somme des estimations stratifiées par évolution(8 040 cas) et origine (4 722 cas) diffère de l'estimation totale du nombre de cas (9 549). 
Tableau 4 : Exhaustivité de la surveillance des intoxications médicamenteuses (CAPM) et des RHR, stratifiée par année, province, sexe, âge, origine, circonstances de l'intoxication et évolution, Tanger-Tétouan-Al Hoceima, Maroc, 2014-2016

\begin{tabular}{|c|c|c|c|c|c|c|c|}
\hline & \multicolumn{5}{|c|}{ Nombre de cas } & \multicolumn{2}{|c|}{ Exhaustivité \% } \\
\hline & CAPM & RHR & $\begin{array}{l}\text { CAPM } \\
+ \text { RHR } \\
\end{array}$ & Communs & Estimé & CAPM & RHR \\
\hline \multicolumn{8}{|c|}{ Année } \\
\hline 2014 & $249(29,8)$ & $286(32,1)$ & $518(31,4)$ & 17 & 4189 & 5,94 & 6,83 \\
\hline 2015 & $292(35,0)$ & $334(37,4)$ & $618(37,5)$ & 8 & 12191 & 2,39 & 27,40 \\
\hline 2016 & $294(35,2)$ & $272(30,5)$ & $513(31,1)$ & 53 & 1509 & 19,48 & 18,02 \\
\hline Total & $835(100)$ & $892(100)$ & $1649(100)$ & 78 & 9549 & 8,74 & 9,34 \\
\hline \multicolumn{8}{|c|}{ Province } \\
\hline Al Hoceima & $49(5,9)$ & $15(1,7)$ & $64(3,9)$ & 0 & 799 & 6,13 & 1,88 \\
\hline Chefchaouen & $121(14,5)$ & $129(14,5)$ & $223(13,5)$ & 27 & 578 & 20,93 & 22,32 \\
\hline Larache & $107(12,8)$ & $153(17,2)$ & $253(15,3)$ & 7 & 2339 & 4,57 & 6,54 \\
\hline M'diq-Fnideq & $52(6,2)$ & $58(6,5)$ & $105(6,4)$ & 5 & 603 & 8,62 & 9,62 \\
\hline Ouezzane & $27(3,2)$ & $20(2,2)$ & $46(2,8)$ & 1 & 540 & 5 & 3,70 \\
\hline Tanger-Assilah & $220(26,4)$ & $260(29,1)$ & $480(29,1)$ & 0 & 57680 & 0,38 & 0,45 \\
\hline Tétouan & $259(31,0)$ & $257(28,8)$ & $478(29,0)$ & 38 & 1752 & 14,78 & 14,67 \\
\hline Total & $835(100)$ & $892(100)$ & $1649(100)$ & 78 & 9549 & 8,74 & 9,34 \\
\hline \multicolumn{8}{|l|}{ Sexe } \\
\hline Femme & $564(68,9)$ & $659(77,3)$ & $1165(73,1)$ & 58 & 6408 & 8,80 & 10,28 \\
\hline Homme & $255(31,1)$ & $194(22,7)$ & $429(26,9)$ & 20 & 2474 & 10,30 & 7,84 \\
\hline Total & $819(100)$ & $853(100)$ & $1594(100)$ & 78 & 8957 & 9,14 & 9,52 \\
\hline \multicolumn{8}{|c|}{ Age } \\
\hline Nouveau-né & - & $1(0,2)$ & $1(0,1)$ & 0 & 1 & 0 & 100 \\
\hline Nourrisson & $19(2,4)$ & $3(0,4)$ & $22(1,5)$ & 0 & 79 & 24,05 & 3,80 \\
\hline Bébé marcheur & $226(28,3)$ & $37(5,4)$ & $255(18,0)$ & 8 & 1045 & 21,63 & 3,54 \\
\hline Enfant & $72(9,0)$ & $45(6,6)$ & $114(8,1)$ & 3 & 1080 & 4,16 & 4,17 \\
\hline Adolescent & $146(18,3)$ & $154(22,7)$ & $287(20,3)$ & 13 & 1730 & 8,44 & 8,90 \\
\hline Adulte & $334(41,9)$ & $438(64,4)$ & $734(51,8)$ & 38 & 3850 & 8,67 & 11,37 \\
\hline Personne âgée & $1(0,1)$ & $2(0,3)$ & $3(0,2)$ & 0 & 5 & 20 & 40 \\
\hline Total & $798(100)$ & $680(100)$ & $1416(100)$ & 62 & 8752 & 9,12 & 7,76 \\
\hline \multicolumn{8}{|c|}{ Origine } \\
\hline Rural & $61(8,3)$ & $59(21,3)$ & $111(11,5)$ & 9 & 400 & 15,25 & 14,75 \\
\hline Urbain & $672(91,7)$ & $218(78,7)$ & $856(88,5)$ & 34 & 4309 & 15,60 & 5,06 \\
\hline Total & $733(100)$ & $277(100)$ & $967(100)$ & 43 & 4722 & 15,52 & 5,87 \\
\hline \multicolumn{8}{|c|}{ Circonstances de l'intoxication } \\
\hline Accidentelles & $461(58,4)$ & $797(91,7)$ & $1193(75,5)$ & 65 & 5653 & 8,15 & 14,10 \\
\hline Volontaires & $329(41,6)$ & $72(8,3)$ & $388(24,5)$ & 13 & 1822 & 18,06 & 3,95 \\
\hline Total & $790(100)$ & $869(100)$ & $1581(100)$ & 78 & 8801 & 8,98 & 9,87 \\
\hline \multicolumn{8}{|c|}{ Evolution } \\
\hline Décès & $6(0,9)$ & $1(0,1)$ & $7(0,5)$ & 0 & 13 & 46,15 & 7,69 \\
\hline Favorable & $635(99,1)$ & $877(99,9)$ & $1442(99,5)$ & 70 & 7956 & 7,98 & 11,02 \\
\hline Total & $641(100)$ & $878(100)$ & $1449(100)$ & 70 & 8040 & 7,97 & 10,92 \\
\hline
\end{tabular}




\section{Discussion}

Dans notre étude, on a appliqué la méthode capture-recapture à deux sources décrite par Sekar et Deming (1949) qui permet, sous certaines conditions, d'estimer le nombre de cas non identifiés par les deuxsources et le taux d'exhaustivité de chaque source.

La recherche des cas communs entre les deux sources a permis d'identifier 78 doublons.Nous avons tenu compte de la similitude de la date d'intoxication, du sexe et de l'âge de l'intoxiqué pour considérer ces cas comme des doublons.

Il est rare de disposer d'un seul identifiant qui permet de détecter les cas communs. Une surestimation de cas communs induit une sous-estimation du nombre total de cas, et inversement (Mastro T.D. et al, 1994 ; Seber GA.et al,2000).

Dans notre étude, les variables choisies pour l'identification des doublons étaientspécifiques et minimisent le risque d'identification de faux positifs. De plus, la vérification manuelle des cas prend en compte les erreurs de transcription.

L'application de la méthode capture-recapture a permis d'estimer le nombre total de cas d'intoxications médicamenteuses identifiés entre 2014 et 2016 au niveau de la région d'étude à 9 549(IC à 95\%: 8 199-10 900). Les résultats ont montré une exhaustivité faiblepour la source CAPM et la source RHR, soit 8,74\%et9,34\% respectivement.Le regroupement des deux systèmes a permis de recenser $17,26 \%$ du nombre total de cas.

Le taux d'exhaustivité dela surveillance des intoxications médicamenteusesdans la région d'étude resteplus faible que celui trouvé en France pour d'autres maladies à déclaration obligatoire tellesque les épidémiesde grippe et les infections à méningocoque. (Hubert B. et al ; 1993 ; Hubert B.et al, 2015).

Cette sous-déclaration peut être expliquée par le manque de connaissances concernant la liste des maladies à déclaration obligatoire, les modalités de déclaration ou les personnes auxquelles les données en question doivent être communiquées et chez les médecins informés de l'obligation de déclarer,le manque de temps etla croyance qu'un grand nombre de maladies à déclaration obligatoire ne sont pas suffisamment importantes pour justifier les efforts qu'exige leur déclaration (Herida M.et al,2008)

L'utilisation des registres des hôpitaux comme deuxième source de données ne permet de collecter que les cas hospitalisés, alors qu'il y a des cas qui peuvent être non hospitalisés.

L'analyse stratifiée sur différents variables susceptibles d'entraînerune hétérogénéité de capture des cas, l'année de déclaration, la province, lesexe et l'âge des patients, les circonstances de l'incident ne montre pas de différence entre la somme des estimations pour chaque strate et l'estimation totale du 
nombre de cas. Chacune des sommes des estimations stratifiées est comprise dans l'Intervalle de Confiance 95\%de l'estimation globale. Toutefois, la somme des estimations stratifiées par évolution et origine s'écarte de l'estimation totale du nombre de cas.

\section{Conclusion}

Des efforts doivent être fournis pour améliorer l'exhaustivité de la déclaration obligatoire des intoxications médicamenteuses dans la région d'étude. Une meilleure sensibilisation des médecins à la déclaration s'avère nécessaire afin de renforcer le système de surveillance des intoxications au Maroc.

\section{References:}

1. Bettaieb J., Bellali H., Ben Alaya N., Mrabet A., KouniChahed M. Évaluation de la surveillance des infections invasives à méningocoques à Tunis par la méthode de capture-recapture. Santé Publique, 2013, 25(5), 609-615.

2. Campèse C., Che D. Evaluation quantitative du système de surveillance des légionelloses en France en 2010. Ed. Saint-Maurice : Institut de veille sanitaire, 2012, 40 p.

3. Chapman D G.Some properties of the hypergeometric distribution with applications to zoological sample censuses. Berkeley, University of California Press, 1951.

4. Cormack R.M. The statistics of capture-recapture methods. Oceanography and Marine Biology: An Annual Review, 1968, 6, 455506.

5. Herida M., Pillonel J., le Strat Y., Desenclos J-C., Saura C. Évaluation du dispositif des maladies à déclaration obligatoire en France : connaissances, attitudes et pratiques des médecins et des biologistes, 2005. Bull EpidémiolHebd. 2008, 51-52, 503-507.

6. Hmimou R., Rhalem N., Chaoui H., Semlali I., Aghandous R., Benlarabi S., Badri M., Soulaymani-Bencheikh R. Rapport général 2015 de toxicovigilance. Toxicologie Maroc, 2015, $\mathrm{N}^{\circ} 27,4^{\text {ème }}$ Trimestre, 3-6.

7. Hubert B., Desenclos J-C. Evaluation de l'exhaustivité et de la représentativité d'un système de surveillance par la méthode de capture-recapture. Application à la surveillance des infections à méningocoque en France en 1989 et 1990. Revue d'Epidémiologie et de Santé Publique, 1993, 41(3), 241-249.

8. Hubert B., Fortin N., Ollivier R., Barataud D. et les réseaux de surveillance de la grippe dans les Pays de la Loire. La surveillance des épidémies de grippe à un niveau régional. exemple de l'épidémie de 
l'hiver 2014-2015 dans les pays de la Loire, France. Bull EpidémiolHebd., 2015, 32-33, 604-611.

9. International Working Group for Disease Monitoring and Forecasting. Capture-Recapture and Multiple-Record Systems Estimation I: History and Theoretical Development.Am J Epidemiol. 1995, 142(10), 1047-1058.

10. Khattabi A., Rhalem N., Soulaymani-Bencheikh R. Centre Anti Poison et de Pharmacovigilance du Maroc : naissance, défis et promesses. Toxicologie Maroc, 2009, $\mathrm{N}^{\circ}$ 1, 3-7.

11. Lebel G., DubéM.Analyse descriptive des appels au Centre antipoison du Québec de 2008 à 2014. Bulletin d'Information en Santé Environnementale, Juin 2015, 1-13.

12. Mathieu-Nolf M. Rapport Annuel 2002 Centre Antipoison de Lille. 2002.

13. Mastro TD., Kitayaporn D., Weniger BG., Vanichseni S., Laosunthorn V., Uneklabh T., Uneklabh C., Choopanya K., Limpakarnjanarat K. Estimating the number of HIV-infected injection drug users in Bangkok: a capture--recapture method. Am J Public Health, 1994, 84(7), 1094-1099.

14. Perrocheau A.,Levy-Bruhl D.Les infections à méningocoques en France en 1998 et 1999. Bull EpidémiolHebd., 2000, 51, 227-229.

15. Seber G.A.F. The Effects of Trap Response on Tag Recapture Estimates. Biometrics, 1970, 26 (1), 13-22

16. Seber GA., Huakau JT., Simmons D. Capture-recapture, epidemiology, and list mismatches: two lists. Biometrics, 2000, 56(4) 1227-32.

17. Sekar C.C., Deming W.E.On a Method of Estimating Birth and Death Rates and the Extent of Registration. Journal of the American Statistical Association, 1949, 44(245), 101-115.

18. Seydaoglu G., Satar S., Alparslan N. Frequency and mortality risk factors of acute adult poisoning in Adana, Turkey, 1997-2002. Mt Sinai J Med. 2005, 72(6), 393-401.

19. Van Hest R. Capture-recapture Methods in Surveillance of Tuberculosis and Other Infectious Diseases. Thesis Erasmus MC, 2007, University Medical Center Rotterdam. 